IFT/2007-07-25

\title{
A note on the topological order of noncommutative Hall fluids
}

\author{
J.L.F. BARbón And D. Gerber \\ Instituto de Física Teórica IFT UAM/CSIC \\ Facultad de Ciencias C-XVI \\ C.U. Cantoblanco, E-28049 Madrid, Spain \\ jose.barbon@uam.es daniel.gerber@estudiante.uam.es
}

\begin{abstract}
We evaluate the ground state degeneracy of noncommutative Chern-Simons models on the twotorus, a quantity that is interpreted as the "topological order" of associated phases of Hall fluids. We define the noncommutative theory via T-duality from an ordinary Chern-Simons model with non-abelian 't Hooft magnetic fluxes. Motivated by this T-duality, we propose a discrete family of noncommutative, non-abelian fluid models, arising as a natural generalization of the standard noncommutative Chern-Simons effective models. We compute the topological order for these universality classes, and comment on their possible microscopic interpretation.
\end{abstract}

November 9, 2018 


\section{Introduction}

Hydrodynamical models of quantum Hall fluids contain a universal Chern-Simons term

$$
S_{\mathrm{eff}}=\frac{k}{4 \pi} \int a \wedge d a+\ldots
$$

that dominates the long-distance dynamics $[1-6]$. The description of the fluid density perturbations in terms of a $2+1$ dimensional gauge field is characteristic of superfluids, the $U(1)$ gauge symmetry being a remnant of the underlying quantum permutation symmetry of identical particles forming the fluid [7]. The emergence of a particular Chern-Simons term with nonvanishing integral coupling, $k$, is related to the broken parity and time reversal invariance by the magnetic field and to the incompressibility of the fluid, so that density perturbations have a gap proportional to $\sqrt{k}$. In the simple phases such as the Laughlin series [8], $k$ is an odd integer equal to $1 / \nu$ with $\nu$ the filling fraction. All the universal properties of those Hall fluids, such as the spectrum of edge excitations, quasiparticle statistics and topological order parameters, are controlled by the single integer $k$. One particularly novel property of these fluids is a new kind of vacuum order, the so-called topological order: the structure of the Hilbert space is partially determined by the spatial topology of the fluid. In terms of the effective theory (11), the topological order is given by the finite dimension of the Hilbert space: $\mathcal{D}_{g} \equiv \operatorname{dim} \mathcal{H}\left(\Sigma_{g}\right)=k^{g}$ on a Riemann surface of genus $g$. For a general review and references on the physics of the Hall effect see for example [9].

For more general Hall phases, there exist some standard generalizations of (1), such as the multi-component abelian fluid models (c.f. [10-15]),

$$
S_{\mathrm{eff}}=\sum_{I, J} \frac{K_{I J}}{4 \pi} \int a_{I} \wedge d a_{J}+\ldots
$$

with $K_{I J}$ an appropriate matrix of integers that characterizes completely the Hall fluid, including the spectrum and statistics of quasiparticles and topological orders. For example, the topological order on a genus- $g$ Riemann surface is given by $\mathcal{D}_{g}=|\operatorname{det}(K)|^{g}$. The various components of the fluid could be rooted on specific properties of the system, such as the case of multilayered electrons, or the relevance of spin degrees of freedom. In other cases, they just provide a phenomenological parametrization of the microscopic dynamics. Some of these models with fluid $U(1)^{\otimes n}$ symmetry might admit a more accurate description in terms of non-abelian ChernSimons topological theories, leading to non-abelian statistics of quasiparticles $[16,17]$.

More recently, it was proposed in [18] that a more accurate version of the hydrodynamics, capable of keeping track of the 'granular' character of the electrons, is provided by the realization of the quantum statistics symmetry of $N$ electrons, $S_{N}$, as the Weyl subgroup of the unitary group $U(N)$. In this scheme, one promotes the electrons' coordinates to the eigenvalues of two $N \times N$ hermitian matrices, and declares $U(N)$ to be a gauge symmetry, so that the number of gauge-invariant degrees of freedom remains the same (the $N$ 'electron positions'). The Lagrangian fluid description then yields the noncommutative generalization of (1),

$$
S_{\mathrm{eff}}=\frac{k}{4 \pi} \int\left(A \wedge_{\star} d A+\frac{2 i}{3} A \wedge_{\star} A \wedge_{\star} A\right),
$$

where the Moyal exterior product is defined by $\alpha \wedge_{\star} \beta=\alpha_{i} \star \beta_{j} d x^{i} \wedge d x^{j}$ and the ordinary Moyal product:

$$
x_{1} \star x_{2}=x_{1} x_{2}+i \theta / 2, \quad\left[x_{1}, x_{2}\right]_{\star}=i \theta .
$$


The elementary area of nonlocality is set by the uniform particle density of the fluid, $\theta=$ $\left(2 \pi \rho_{e}\right)^{-1}$, in some equilibrium configuration. A first satisfactory consequence of this effective discreteness of space is that the level (and thus the inverse filling fraction) must be quantized, due to the non-connectedness of the group of gauge transformations; notably, this holds even for the $U(1)$ theory on $\mathbf{R}_{\theta}^{2}[19,20]$ (see also [21]).

The noncommutative $U(1)_{\star}$ gauge theory at hand is naturally defined on noncommutative deformations of standard manifolds, but note that non-compact cases like $\mathbf{R}_{\theta}^{2}$ imply a thermodynamic limit in the number of electrons, $N \rightarrow \infty$. Various finite $N$ versions of (3) have been proposed as finite-matrix truncations, appropriate for the description of Hall droplets [22-25] and variations thereof [26-28]. Here we consider a noncommutative torus $\mathbf{T}_{\theta}^{2}$ of area $L^{2}$, and define for convenience the dimensionless noncommutativity parameter $\Theta \equiv 2 \pi \theta / L^{2}$. When this parameter is a rational number, the resulting theory has special properties. This is indeed our case, since $\Theta=1 / N$, the inverse of the number of electrons.

The organization of the paper is as follows. In the rest of this introduction, we argue that the topological order is insensitive to a noncommutative deformation (1.1), and show this more precisely for tori using T-duality (1.2). In section 2, we propose a family of slightly generalized toroidal Hall fluids, motivated by the T-duality properties of (3). The asymmetric levels in these models must obey a consistency condition; section 2.1 describes how the latter arises, and section 2.2 uses it in computing the topological order on a torus. Section 3 proposes some microscopic interpretations.

\subsection{Wen's topological order and noncommutativity}

The main physical input of (3) is the noncommutative generalization of the fluid gauge symmetry. In this regard, one important property of this generalization is a certain tension with the phenomenological multifluid models. $U(1)_{\star}^{\otimes n}$ gauge invariance of (2) seems to require a purely diagonal $K_{I J}$ matrix, due to the non-commuting character of the Moyal product. This is the case even when all the density parameters, $\theta$, are equal to one another. Hence, if we demand polynomial interactions in the Moyal product, any non-abelian enhancement of the $U(1)_{\star}^{\otimes n}$ multifluid gauge symmetry is necessarily a diagonal product of $U\left(n_{I}\right)_{\star}$ groups. Without loss of generality, we can then concentrate on the case of a simple $U(n)_{\star}$ gauge factor, i.e. we have the action (3), where now $A$ is a noncommutative one-form taking values on the Lie algebra $u(n) \equiv \operatorname{Lie}[U(n)]$.

The second important property of (3) is its apparent independence of the noncommutative deformation parameter $\theta$. Despite the infinite tower of nonlinear corrections embodied in the Moyal product, the noncommutative model on $\mathbf{R}_{\theta}^{2}$ ends up being classically equivalent to (1) under a Seiberg-Witten map (c.f. $[29,30]$ ). This suggests that (3) contains just the same topological information as (1). It also implies that a noncommutative multifluid theory with general $K_{I J}$ matrix should exist, albeit with a degree of nonlocality that cannot be simply parameterized by polynomials in the Moyal product. A more precise statement can be put forward for the model defined on the noncommutative torus, $\mathbf{T}_{\theta}^{2}$. In canonical quantization, choosing temporal gauge $A_{0}=0$, we are left with the purely abelian 'kinetic term' in (3) for the spatial components of the gauge field, plus the Gauss constraint

$$
\partial_{1} A_{2}-\partial_{2} A_{1}+i\left[A_{1}, A_{2}\right]_{\star}=0
$$


If the gauge field is defined with periodic boundary conditions on $\mathbf{T}_{\theta}^{2}$, the natural ansatz for the solution of (4) is that $A_{i} d x^{i}$ be a constant one-form over $\mathbf{T}_{\theta}^{2}$. But those are precisely insensitive to the noncommutativity, so that we end up with a moduli space of flat connections independent of $\theta$, i.e. we have the standard moduli space of $U(n)$ flat connections, which can be parameterized locally by angular variables $c^{a}, a=1, \ldots, n$, running in the Cartan torus of $U(n), \mathbf{T}_{\mathrm{C}}$. Dividing by the residual Weyl group symmetry leads to the orbifold

$$
\mathcal{M}=\frac{\mathbf{T}_{\mathrm{C}} \times \mathbf{T}_{\mathrm{C}}}{W_{n}}
$$

with $W_{n}=S_{n}$ acting diagonally on the product of the two tori. We quantize this moduli space by reducing the Chern-Simons action to zero-modes:

$$
S_{\text {flat }}=2 \pi k \int d t \sum_{a=1}^{n} \dot{c}_{1}^{a} c_{2}^{a}
$$

Interpreting the modding by the Weyl group as the statistical constraint for $n$ identical bosons on the torus $\left(c_{1}, c_{2}\right)$ of unit size, this model is isomorphic to $n$ bosons in a Landau level of $k$ states. Hence, the dimension of the Hilbert space (the topological order on the torus) is simply given by the number of ways of distributing the $n$ bosons over $k$ 'orbitals':

$$
\mathcal{D}_{1}=\operatorname{dim} \mathcal{H}\left(\mathbf{T}_{\theta}^{2}\right)=\frac{(n+k-1) !}{n !(k-1) !} .
$$

Notice that the result does not depend on $\theta$, and differs in general from other natural models with $n$ fluid components, such as the Jain hierarchical scheme for filling fractions $\nu=n / k$ and $k=2 n s+1, s \in \mathbf{Z}$. In this case, the topological order is given by $\mathcal{D}_{1}=k$. Hence, the result (6) seems to pose a very stringent limitation to any model that combines enhanced gauge symmetry and noncommutativity.

\subsection{T-duality}

The arguments just presented can be made more rigorous using T-duality 1 : an $S L(2, \mathbf{Z})$ group of discrete transformations between theories of different values of $\Theta$ and different ranks and quantum numbers. Given that $\Theta=1 / N$ is rational in our case, there is a T-duality transformation that maps (3) to a level $\widetilde{k}=k N$ Chern-Simons model with gauge group $U(\tilde{n})$, $\tilde{n}=n N$, and defined on a commutative $(\theta=0)$ two-torus of size $\widetilde{L}=L / N$. If the original $U(n)_{\star}$ model was defined with periodic boundary conditions on $\mathbf{T}_{\theta}^{2}$, the T-dual $U(n N)$ non-abelian (but commutative) model is restricted to $U(n N)$ bundles with first Chern class $c_{1}=n$. Since only the diagonal $U(1)$ subgroup contributes to this Chern class, such bundles can be viewed as $U(1) \times\left(S U(n N) / \mathbf{Z}_{n N}\right)$ bundles with $n$ units of magnetic flux on the $U(1)$ factor and $n$ units of 't Hooft magnetic flux on the $S U(n N) / \mathbf{Z}_{n N}$ subgroup (see [34] for a more detailed exposition).

Notice that the T-dual representation uses an unphysical torus $\widetilde{\mathbf{T}}^{2}$ of length $\widetilde{L}=L / N$. In realistic situations, $N$ is the number of electrons in the fluid and therefore macroscopic, $\widetilde{L}$

\footnotetext{
${ }^{1}$ Also known as Morita equivalence in the mathematical literature [31]. Morita equivalence in general relates among themselves different non-commutative spaces (i.e. $\mathrm{C}^{*}$-algebras), along with -in nice cases like here- all the bundle structures necessary to define an action. For non-commutative tori, these transformations can be thought of as a remnant of the full T-duality of a corresponding string [32] or matrix [33] theory.
} 
being extremely small. The dual model must be regarded as a mathematical tool to define the quantization of the original noncommutative theory. In specifying path integration in the Tdual model, the gauge fields must be restricted to some particular class of (non-trivial) bundles over the torus. To this end, we can consider a three-dimensional manifold $\widetilde{\mathbf{D}}^{3}$ with boundary, $\partial \widetilde{\mathbf{D}}^{3}=\widetilde{\mathbf{T}}^{2}$, over which the bundle extends trivially. Then we can write the dual action as

$$
\widetilde{S}_{\text {eff }}=\frac{\widetilde{k}}{4 \pi} \int_{\widetilde{\mathbf{D}}^{3} \times \mathbf{R}} \widetilde{\operatorname{Tr}}(\widetilde{F}-\widetilde{\Phi}) \wedge(\widetilde{F}-\widetilde{\Phi}),
$$

where $\widetilde{T r}$ denotes the trace in the fundamental representation of $U(\tilde{n})=U(n N)$. In this expression, $\widetilde{\Phi}$ denotes a constant-curvature $U(1)$ field strength, precisely subtracting the $n$ units of magnetic flux in the diagonal $U(1)$ group. Hence, we can regard the model as defined on $U(n N)$ bundles of the form $U(1) \times\left(S U(n N) / \mathbf{Z}_{n N}\right)$, where the $U(1)$ factor has zero curvature and the $S U(n N) / \mathbf{Z}_{n N}$ bundle has $n$ units of 't Hooft magnetic flux through the two torus $\widetilde{\mathbf{T}}^{2}$. After we implement this constraint on the topological structure of the bundles, we can drop the constant background $\widetilde{\Phi}$ from the action (7).

The canonical quantization of this model follows the usual procedure, i.e. we must quantize the moduli space of flat connections. For the particular $U(n N)$ bundles under consideration, the moduli space of flat connections is the direct product of the diagonal $U(1)$ contribution, times the flat connections of the $S U(\tilde{n}) / \mathbf{Z}_{\tilde{n}}$ theory with $n$ units of 't Hooft flux. The latter are the solutions of the equations

$$
\widetilde{U}_{1} \widetilde{U}_{2}=\widetilde{U}_{2} \widetilde{U}_{1} e^{2 \pi i / N},
$$

where $\widetilde{U}_{1}, \widetilde{U}_{2}$ are matrices in $S U(\tilde{n})$. There is an irreducible solution of this equation in $S U(N)$ matrices, given by the standard clock and shift matrices $\Gamma_{1}, \Gamma_{2}$. Then, the general solution of (8) is, up to conjugacy, $\widetilde{U}_{i}=W_{i} \otimes \Gamma_{i}, i=1,2$, with $W_{i}$ a pair of commuting matrices in $S U(n)$. Hence, the required moduli space of flat connections is isomorphic to that of an $S U(n)$ bundle with periodic boundary conditions. Adding back the diagonal $U(1)$ factor, one finds the flat connections of an $U(n)$ gauge model, with reduced dynamics given exactly by (5) (see [34]). From here, (6) follows as in the previous discussion.

We conclude that the independence of the topological order as a function of $\theta$ can be readily obtained -for rational values of $\theta$ - in a rigorous definition of the theory via the T-duality transformation to a twisted non-abelian, but commutative model.

\section{Generalized noncommutative fluids}

We have seen in the previous section that the topological order is a priori insensible to a noncommutative deformation. In this section, we use the quantum definition of the toroidal fluid to propose a natural generalization that behaves discontinuously at $\theta=0$.

One of the basic features of nonabelian noncommutative gauge theories is the automatic promotion of $S U(n)$ gauge groups to full $U(n)_{\star}$ gauge invariance. The Moyal commutator, $\left[A_{1}, A_{2}\right]_{\star}$, responsible for the self-interaction of the gauge fields, is proportional to

$$
\left[T^{a}, T^{b}\right] \cos \left(\frac{1}{2} \theta p_{1} p_{2}^{\prime}\right)+\left\{T^{a}, T^{b}\right\} \sin \left(\frac{1}{2} \theta p_{1} p_{2}^{\prime}\right),
$$

where $T^{a}$ is a basis of generators of the $u(n)$ Lie algebra, and $p, p^{\prime}$ are the momenta entering the vertex. Hence, the diagonal $U(1)$, from the local decomposition $u(n)=u(1) \oplus s u(n)$ of the Lie algebra, remains coupled at generic values of the momenta. 
Something peculiar happens when the theory is formulated on a torus with rational noncommutativity parameter $\Theta$. On a torus we have $p=2 \pi n / L$ and the anticommutator part yields

$$
\left\{T^{a}, T^{b}\right\} \sin \left(\pi \Theta n_{1} n_{2}^{\prime}\right) .
$$

In our case $\Theta=N^{-1}$ and the diagonal $U(1)$ does decouple for momentum modes proportional to the number of electrons, $N$. Therefore, there is an effective position-space torus of size $\widetilde{L}=L / N$, which we shall denote $\widetilde{\mathbf{T}}^{2}$, on which the diagonal $U(1)$ modes decouple completely. In the T-dual picture, this result is obvious, since the commutative $U(n N)$ model is now defined on a torus of size $L / N$, and there is no local coupling of the diagonal $U(1)$ subgroup and the $S U(n N)$ subgroup. This peculiar decoupling of $U(1)$ degrees of freedom in rational theories makes it natural to generalize our definition of a 'noncommutative fluid' to encompass these cases, by allowing different Chern-Simons couplings in the two sectors. The resulting Lagrangian is most easily written in T-dual variables, as a Chern-Simons model on the torus $\widetilde{\mathbf{T}}^{2}$ of size $\widetilde{L}=L / N$ with gauge fields in the Lie algebra of the gauge group $U(1)_{\tilde{\kappa} \tilde{n}} \times S U(\tilde{N})_{\tilde{k}}$, and with $n$ units of 't Hooft flux on the $S U(\tilde{n}) / \mathbf{Z}_{\tilde{n}}$ subgroup. The subscripts on the gauge groups indicate the level, or Chern-Simons coupling, with the following normalization convention: decomposing the dual gauge field

$$
\widetilde{A}=\widetilde{a} \cdot \mathbf{1}_{\tilde{n}}+\widetilde{A}^{\prime}, \quad \widetilde{\operatorname{Tr}}\left(\widetilde{A}^{\prime}\right)=0
$$

into $u(1)$ and $s u(n N)$ parts, the action reads

$$
\widetilde{S}_{\mathrm{eff}}=\frac{\tilde{\kappa} \tilde{n}}{4 \pi} \int_{\widetilde{\mathbf{T}}^{2} \times \mathbf{R}}(\widetilde{a} \wedge d \widetilde{a})+\frac{\tilde{k}}{4 \pi} \int_{\widetilde{\mathbf{D}}^{3} \times \mathbf{R}} \widetilde{\operatorname{Tr}}\left(\widetilde{F}^{\prime} \wedge \widetilde{F}^{\prime}\right) .
$$

The classical $U(n)_{\star}$ model written in T-dual variables, eq. (7), is obtained in the case $\tilde{\kappa}=\tilde{k}$. Recall that the T-duality map implies $\tilde{n}=n N$ and $\tilde{k}=k N$. Hence, we will find it useful to parametrize the abelian level as $\tilde{\kappa}=\kappa N$.

Going back to the original variables, in terms of Moyal products, we have

$$
S_{\text {eff }}=(\kappa-k) \frac{n N^{2}}{4 \pi} \int_{\widetilde{\mathbf{T}}^{2} \times \mathbf{R}} \widetilde{a} \wedge d \widetilde{a}+\frac{k}{4 \pi} \int_{\mathbf{T}_{\theta}^{2} \times \mathbf{R}} \operatorname{Tr}\left(A \wedge_{\star} d A+\frac{2 i}{3} A \wedge_{\star} A \wedge_{\star} A\right) .
$$

In this expression we have kept the dual notation for the purely abelian term, in order to have a local expression in position space. In the original representation on $\mathbf{T}_{\theta}^{2}$, we must specify to keep only those diagonal $U(1)$ degrees of freedom whose momentum quantum numbers vanish modulo $N$.

The asymmetry between the abelian and non-abelian sectors is measured by the difference $\kappa-k$. For $\kappa=k$, we recover the lagrangian (77) of the previous section. However, different choices of $\kappa$ might be natural depending on particular circumstances. For example, if the original noncommutative gauge field couples to massive degrees of freedom in the adjoint representation, integrating them out induces a shift of the low-energy Chern-Simons level at one loop order. Thus for each bosonic degree of freedom in the adjoint representation the level shifts $k \rightarrow$ $k+n$, whereas the shift is only half as much and negative, $k \rightarrow k-\frac{1}{2} n$ for each real fermionic (Majorana) degree of freedom in the adjoint representation. On the rational noncommutative torus, all these shifts leave intact the diagonal $U(1)$ fields with momenta proportional to $N$ (see [35] for a recent explicit check). Hence, by integrating out massive adjoints we can end up with an effective $\kappa$ that differs from $k$. 
On the other hand, Wilson loop expectation values in an ordinary $S U(n)$ Chern-Simons model are naturally a function of $k+n$, whereas the abelian counterparts lead to simple functions of $k$. In order to have a simple $U(n)$ symmetry action over quantum Wilson lines, it is useful to start with a classical model of the form (10) with $\kappa=k+n$. Hence, we see various instances in which consideration of the generalized fluids (10) might be regarded as natural.

\subsection{Global properties}

What we have said so far only refers to couplings in the Lagrangian. However, the precise definition of the non-abelian fluid models requires further discrete projections, particularly if we wish to recover the $U(n)_{\star}$ model at $\kappa=k$, discussed in the previous section. The global structure of the $U(n)$ group, i.e. $U(n)=(U(1) \times S U(n)) / \mathbf{Z}_{n}$ implies that the flat connections in the diagonal $U(1)$ are correlated with those of the non-abelian factor by the modding by the centre of $S U(n)$. That is, the $\mathbf{Z}_{n}$ redundancy $U=u U^{\prime}=u z \cdot z^{-1} U^{\prime}$, with $u \in U(1), U^{\prime} \in S U(n)$ and $z \in \mathbf{Z}_{n}$, in the decomposition of an arbitrary $U(n)$ matrix, is treated as a discrete gauge symmetry and the theory is projected onto the $\mathbf{Z}_{n}$-invariant sector.

More specifically, the $\mathbf{Z}_{n}$ group acts by 'large' gauge transformations on the $S U(\tilde{n}) / \mathbf{Z}_{\tilde{n}}$ factor. For Chern-Simons theories, it is enough to specify this action at the level of flat connections on the torus. On a bundle with $n$ units of 't Hooft flux, flat connections are specified by the holonomies $\widetilde{U}_{1}, \widetilde{U}_{2}$ in the two directions of the torus. As pointed out above, these have the form $\widetilde{U}_{i}=W_{i} \otimes \Gamma_{i}$ with $W_{i}$ a pair of commuting matrices in $S U(n)$ and $\Gamma_{i}$ the (essentially unique) solution of $\Gamma_{1} \Gamma_{2}=\Gamma_{2} \Gamma_{1} \exp (2 \pi i / N)$ in $S U(N)$ matrices. Then, there is a $\mathbf{Z}_{n} \times \mathbf{Z}_{n}$ group of large gauge transformations acting as

$$
W_{j} \rightarrow z_{j} W_{j}, \quad j=1,2,
$$

with $z_{j}=\exp \left(2 \pi i \alpha_{j} / n\right), \alpha_{j} \in \mathbf{Z}$. On the Cartan torus of the $W_{i}$, the group $\mathbf{Z}_{n}$ acts by discrete translations proportional to $\alpha_{j} / n$. The flat holonomies on the abelian diagonal $U(1)$ are given by a pair of phases $w_{j}$, acted upon by the $\mathbf{Z}_{n} \times \mathbf{Z}_{n}$ group as

$$
w_{j} \rightarrow z_{j}^{-1} w_{j}, \quad j=1,2,
$$

so that the total holonomy $w_{j} W_{j}$ remains $\mathbf{Z}_{n}$-invariant.

Upon canonical quantization, wave functionals can be chosen in irreducible representations labelled by the associated characters, which are known as 't Hooft electric flux sectors in ordinary gauge theories. In topological Chern-Simons theories, the flat holonomies along each direction of the torus end up as canonical conjugates of one another. Therefore, only one set of holonomies, and their corresponding set of electric flux sectors, is used to label physical states. Following this procedure, let us conventionally choose $w_{1} \in U(1), W_{1} \in S U(n)$ as the flat holonomies parametrizing the configuration space of the system. The Hilbert space of the level- $\kappa n$ abelian Chern-Simons theory consists of wave functions $\psi_{e_{1}}\left(w_{1}\right)$, partially classified by their representation under $\mathbf{Z}_{n}$, i.e. by the value of the electric flux $e_{1}$, an integer defined modulo $n$ by the transformation:

$$
\psi_{e_{1}}\left(w_{1}\right) \rightarrow \psi_{e_{1}}\left(z_{1}^{-1} w_{1}\right)=\left(z_{1}\right)^{-e_{1}} \psi_{e_{1}}\left(w_{1}\right)
$$

For the non-abelian $S U(\tilde{n}) / \mathbf{Z}_{\tilde{n}}$ sector we have analogous electric flux sectors $e_{1}^{\prime}$,

$$
\Psi_{e_{1}^{\prime}}\left(W_{1}\right) \rightarrow \Psi_{e_{1}^{\prime}}\left(z_{1} W_{1}\right)=\left(z_{1}\right)^{e_{1}^{\prime}} \Psi_{e_{1}^{\prime}}\left(W_{1}\right),
$$


also given as integers modulo $n$. When collecting together the abelian and non-abelian sectors, the projection to the singlet representation of $\mathbf{Z}_{n}$ imposes the constraint

$$
e_{1}=e_{1}^{\prime}(\bmod n)
$$

which effectively reduces the dimension of the Hilbert space by a factor of $n$ with respect to the dimension of the product theory $U(1)_{\kappa n} \times S U(n)_{k}$. An analogous projection must be enforced for the conjugate 'momentum' variables, $w_{2}, W_{2}$. If the latter is consistent with the first projection, we should end up with a reduction of the naive product dimension by a factor of $n^{2}$. In the next section we confirm this counting by a more detailed analysis of the $\mathbf{Z}_{n}$ action in a convenient basis.

The total dimension of the $S U(n)$ Chern-Simons Hilbert space is given by the number of integrable representations of the $S U(n)_{k}$ Kac-Moody algebra. This number can be computed by counting $S U(n)$ Young tableaux with at most $k$ boxes, yielding

$$
\mathcal{D}_{1}\left(S U(n)_{k}\right)=\frac{(n+k-1) !}{(n-1) ! k !}
$$

On the other hand, the level- $\kappa n$ abelian Chern-Simons model has $\kappa n$ states. Hence, we are predicting

$$
\mathcal{D}_{1}(\kappa, n, k)=\frac{\kappa(n+k-1) !}{n ! k !}
$$

as conjectured in [34]. The condition that this generalized topological order be an integer requires that $\kappa=k(\bmod n)$. In the next section we flesh out this constraint as a consistency condition in the quantization procedure.

\subsection{Calculation of the generalized topological order}

In this section we compute the topological order of the generalized fluids, confirming the conjecture (11). As explained in previous sections, it suffices to quantize the space of naive zero modes of a Chern-Simons model

$$
\frac{U(1)_{\kappa n} \times S U(n)_{k}}{\mathbf{Z}_{n}}
$$

where the group $\mathbf{Z}_{n}$ is interpreted as a discrete gauge symmetry. The reduced configuration space to be quantized consists of the flat holonomies, with associated connections parameterizing the moduli space

$$
\frac{\mathbf{T}_{\mathrm{C}} \times \mathbf{T}_{\mathrm{C}}}{\left(\mathbf{Z}_{n} \times \mathbf{Z}_{n}\right) \times W_{n}}
$$

As before, the two $n$-tori in the numerator arise from the two cycles of the spatial torus. We parameterize these holonomies by diagonal elements $C^{0}$ and $\mathbf{C}$ in $u(n)=u(1) \oplus s u(n)$, with the identifications

$$
C_{i}^{0} \sim C_{i}^{0}+1, \quad \text { and } \quad \mathbf{C}_{i} \sim \mathbf{C}_{i}+\alpha, \quad(i=1,2)
$$

for any (co-)root $\alpha \in \Lambda_{R}$.

The residual Weyl symmetry comes from constant gauge transformations $g(x)=g_{\Gamma} \in S U(n)$ acting by conjugation on both holonomies simultaneously:

$$
W_{n} \ni \Gamma: \quad\left(\mathbf{C}_{1}, \mathbf{C}_{2}\right) \longrightarrow\left(\Gamma^{-1}\left(\mathbf{C}_{1}\right), \Gamma^{-1}\left(\mathbf{C}_{2}\right)\right) .
$$


The division by $\mathbf{Z}_{n}$ accounts for the freedom in lifting a path from $U(n)$ to $U(1) \times S U(n)$. A generator of this group acts, on each $n$-torus separately, by a translation

$$
\mathbf{Z}_{n} \ni \mathbf{T}_{i}^{(j)}: \quad\left(C_{i}^{0}, \mathbf{C}_{i}\right) \longrightarrow\left(C_{i}^{0}-\frac{j}{n}, \mathbf{C}_{i}-\mathbf{w}_{j}\right) \quad i=1,2
$$

for some integer $j$ relatively prime to $n$. For definiteness, we use the trace in the fundamental representation $\langle\cdot, \cdot\rangle \equiv \operatorname{Tr}(\cdot, \cdot)$ to identify $u(n)$ with its dual. The fundamental weights $\mathbf{w}_{j}, j=$ $1, . ., n-1$ are defined as the basis of $s u(n)$ dual to the roots $\alpha_{j} \equiv \operatorname{diag}\left(0, . ., 1_{j},-1_{j+1}, . ., 0\right)$. Note that $r \mathbf{w}_{j} \in \Lambda_{R}$ only for $r$ a multiple of $n$.

In fact the levels play the role of two scaling factors, for the scalar product on $u(1)$ and for the Killing form. It will be convenient to decompose $\mathbf{C}_{1}=C_{1}^{a} \alpha_{a}$ in terms of roots, and $\mathbf{C}_{2}=C_{2}^{a} \mathbf{w}_{a}$ in terms of weights, to have simply $\left\langle\dot{\mathbf{C}}_{1}, \mathbf{C}_{2}\right\rangle=\sum_{a=1}^{n-1} \dot{C}_{1}^{a} C_{2}^{a}$. The reduced action

$$
S_{\text {flat }}=2 \pi \kappa n \int d t \dot{C}_{1}^{0} C_{2}^{0}+2 \pi k \int d t \sum_{a=1}^{n-1} \dot{C}_{1}^{a} C_{2}^{a} .
$$

gives canonical commutation relations

$$
\left[C_{1}^{0}, C_{2}^{0}\right]=\frac{i}{2 \pi \kappa n} \quad\left[C_{1}^{a}, C_{2}^{b}\right]=\frac{i \delta^{a b}}{2 \pi k}
$$

which fit nicely with the product structure in the numerator of the orbifold (12) 2 . It allows to construct the Hilbert space starting with functions in one set of $n$ variables, say $C_{1}^{0}, C_{1}^{a}$, which may be thought of as "positions", and represent in the standard way the conjugate variables, i.e. the 'momenta' $C_{2}^{0}, C_{2}^{a}$, as derivative operators. These wave functions are linear superpositions, $\Psi\left(C_{1}^{0}, \mathbf{C}_{1}\right)=\sum_{(m, \mathbf{M}) \in \mathbf{Z} \times \Lambda_{w}} \Psi_{m, \mathbf{M}} \chi_{m, \mathbf{M}}$, of characters

$$
\chi_{m, \mathbf{M}} \equiv \exp 2 \pi i\left(m C_{1}^{0}+\left\langle\mathbf{M}, \mathbf{C}_{1}\right\rangle\right)
$$

labeled by the abelian and non-abelian weights $m$ and $\mathbf{M}$. Representing the c.c.r. on this space leads to the identifications $m \sim \kappa n C_{2}^{0}$ and $\mathbf{M} \sim k \mathbf{C}_{\mathbf{2}}$. First, this furnishes an alternative interpretation for the characters, as delta functions in the conjugate variables

$$
\chi_{m, \mathbf{M}}\left(C_{2}^{a}\right)=\delta\left(\kappa n C_{2}^{0}-m\right) \delta\left(k \mathbf{C}_{2}-\mathbf{M}\right) .
$$

Second, the total Hilbert space $\hat{\mathcal{H}}$-i.e. the result of quantizing $\mathbf{T}_{\mathrm{C}} \times \mathbf{T}_{\mathrm{C}} /\left(\mathbf{Z}_{n} \times \mathbf{Z}_{n}\right)$ - is effectively finite dimensional and consists of equivalence classes of wave functions labeled by $(m, \mathbf{M}) \in$ $\mathbf{Z}_{\kappa n} \times \Lambda_{w} / k \Lambda_{R}$. Now, the modding of $\hat{\mathcal{H}}$ by the Weyl group and $\mathbf{Z}_{n}$ is to be obtained from their actions (13) and 14) on the underlying phase space (12). An element $\Gamma \in \mathbf{W}_{n}$ sends $\chi_{m, \mathbf{M}}$ to $\chi_{m, \Gamma(\mathbf{M})}$, consistently in both representations (16) and (17) . Similarly, one easily deduces the effect of $\mathbf{Z}_{n}$, for holonomies number one and number two. The relevant actions on wave

\footnotetext{
${ }^{2}$ We shall use a 'bosonic' representation for the wave functions, in which the Weyl group acts as a statistical group on an effective bosonic Hilbert space. A more standard basis used in the literature, which duly incorporates the quantum shift $k \rightarrow k+n$ of the non-abelian theory, involves a 'fermionic' action of the Weyl group (c.f. [36-38]. For the purposes of this note, the bosonic representation as used in [39] [40], is actually more convenient.
} 
functions are

$$
\begin{aligned}
\Gamma: & \Psi_{m, \mathbf{M}} \longrightarrow \Psi_{m, \Gamma^{-1}(\mathbf{M})} \\
T_{1}^{(j)}: & \Psi_{m, \mathbf{M}} \longrightarrow \Psi_{m, \mathbf{M}} \exp 2 \pi i\left(-\frac{m j}{n}-<\mathbf{M}, \mathbf{w}_{j}>\right) \\
T_{2}^{(j)}: & \Psi_{m, \mathbf{M}} \longrightarrow \Psi_{m-j \kappa, \mathbf{M}-k \mathbf{w}_{j}} .
\end{aligned}
$$

Computing their commutators gives

$$
\begin{aligned}
{\left[T_{1}^{(j)} \Gamma\left(T_{1}^{(j)}\right)^{-1} \Gamma^{-1}\right] \Psi_{m, \mathbf{M}} } & =\Psi_{m, \mathbf{M}} \exp 2 \pi i<\Gamma(\mathbf{M})-\mathbf{M}, \mathbf{w}_{j}> \\
& =\Psi_{m, \mathbf{M}} \\
{\left[T_{2}^{(j)} \Gamma\left(T_{2}^{(j)}\right)^{-1} \Gamma^{-1}\right] \Psi_{m, \mathbf{M}} } & =\Psi_{m, \mathbf{M}+k \mathbf{w}_{j}-\Gamma\left(k \mathbf{w}_{j}\right)} \\
& \sim \Psi_{m, \mathbf{M}}
\end{aligned}
$$

using the fact that $\Gamma(\mathbf{w})-\mathbf{w} \in \Lambda_{R}$ for any $\mathbf{w} \in \Lambda_{w}$, and

$$
\left[T_{1}^{(j)} T_{2}^{(l)}\left(T_{1}^{(j)}\right)^{-1}\left(T_{2}^{(l)}\right)^{-1}\right]=\exp -2 \pi i\left(\kappa \frac{j l}{n}+<k \mathbf{w}_{l}, \mathbf{w}_{j}>\right)=\exp 2 \pi i \frac{j l}{n}(k-\kappa)
$$

as the scalar products of the fundamental weights are $\left\langle w_{j}, w_{l}\right\rangle=\min (j, l)-j l / n$ (the inverse of the Cartan matrix).

Thus, we find that, in order to obtain a non-trivial quantum theory (i.e. $\operatorname{dim}(\mathcal{H}) \neq 0$ ), the abelian and non-abelian levels must obey the compatibility condition

$$
\kappa=k+s n
$$

with $s \in \mathbf{Z}$.

In this case, the modding by the denominator in (12) amounts in fact, as far as its effect on $\hat{\mathcal{H}}$ is concerned, to a faithful action of $\mathbf{Z}_{n} \times \mathbf{Z}_{n} \times W_{n}$. In particular, the topological order of the general model can be written as

$$
\mathcal{D}_{1}(\kappa, k, n)=\frac{\mathcal{D}_{1}\left(U(1)_{\kappa n}\right) \cdot \mathcal{D}_{1}\left(S U(n)_{k}\right)}{n^{2}},
$$

and using for calibration its value in the known cases of $U(1)$ and $U(n)$, one has

$$
\mathcal{D}_{1}(\kappa, k, n)=\frac{\kappa \cdot(n+k-1) !}{n ! k !}=\mathcal{D}_{1}\left(U(n)_{k}\right)+s \mathcal{D}_{1}\left(S U(n)_{k}\right) .
$$

Incidentally, this relation also provides an easy way to compute $\mathcal{D}_{1}\left(S U(n)_{k}\right)$.

\section{Discussion}

Noncommutative Chern-Simons models for Hall fluids show little or no dependence on the deformation parameter $\theta$. In particular, the quantum structure of the theory on a compact two-torus is explicitly independent of the rational noncommutativity parameter $\Theta$, a conclusion that follows from careful consideration of the T-duality symmetry. In this note, we have argued 
that the assumption of a noncommutative effective action does impose some constraints on the universality classes of nonabelian fluids, in the form of discrete selection rules.

At the classical level, the statistical gauge symmetry is restricted to be a diagonal product of $U(n)_{\star}$ gauge groups. In particular, multifluid models of the form (2) with non-diagonal $K$ matrix do not admit a polynomial action in Moyal products. Instead, any non-abelian statistics group with an $S U(n)$ factor is forced to combine with a diagonal $U(1)$ into a full $U(n)_{\star}$ gauge group. At the quantum level, the rationality of the noncommutative theory on the torus allows a certain relaxation of this rule, selecting universality classes characterized by groups $\left[U(1)_{\kappa n} \times S U(n)\right] / \mathbf{Z}_{n}$, where the abelian level is restricted by the selection rule $\kappa=k(\bmod n)$.

Among the proposed universality classes, the case $\kappa=k+n$ is especially interesting. For this choice of abelian level, correlation functions of Wilson-line operators have a uniform dependence on the combination $k+n$, suggesting a quantum realization of the $U(n)$ gauge symmetry (c.f. [41]). The associated WZW model of edge currents is also the standard definition of the $u(n)$ Kac-Moody algebra [42]. Finally, this case yields a natural generalization of the 'parton construction' of Hall fluids with nonabelian statistics in [39], to which the concluding remarks are devoted.

Following [39], we can heuristically construct hydrodynamical models with non-abelian statistics by splitting the electron into partons, that are subsequently bound together into full electrons by means of a non-abelian gauge field. The construction presented explicitly in [39] refers in our notation to the case $n=2$, generating fluids with topological order $(k+1)(k+2) / 2$ and wave function $\mathcal{X}_{1}\left(\mathcal{X}_{k}\right)^{2}$, with $\mathcal{X}_{k}$ the standard wave function for $k$ filled Landau levels.

The generalization to fluids with wave function $\mathcal{X}_{1}\left(\mathcal{X}_{k}\right)^{n}$ and $U(n)$ quantum statistics proceeds as follows. We split the electron into $n+1$ partons. The first $n$ partons, with fractional electric charge $q_{s}=1 /(k+n)$, are bound by an $S U(n)$ gauge field into a baryon-like configuration, which is then bound to the remaining parton by the diagonal $U(1)$ gauge group. The second-quantized formalism at the parton level includes field operators $\Phi_{(k)}$ in the fundamental representation of $S U(n)$ and filling $k$ Landau levels, together with field operators $\phi_{(1)}$ with unit Landau multiplicity and charged only under the diagonal $U(1)$ group (since the partons are regarded as fermions, we require $n$ to be an even integer, for the electron bound state to remain a fermion). The electric charge of the remaining parton $q_{d}=k /(k+n)$ ensures that $q_{d}+n q_{s}=1$, the electron charge. The resulting Hamiltonian reads

$$
H_{\text {parton }}=\frac{1}{2 \mu} \phi_{(1)}^{\dagger}\left(-i \nabla-q_{d} \mathbf{A}_{\mathrm{em}}+\operatorname{Tr} \mathbf{A}\right)^{2} \phi_{(1)}+\frac{1}{2 \mu} \Phi_{(k)}^{\dagger}\left(-i \nabla-q_{s} \mathbf{A}_{\mathrm{em}}-\mathbf{A}\right)^{2} \Phi_{(k)}
$$

In this expression, $\mathbf{A}$ denotes the $U(n)$ gauge field, $\operatorname{Tr} \mathbf{A} / n$ is the diagonal $U(1)$ gauge field, and $\mathbf{A}_{\mathrm{em}}$ is the external electromagnetic field. Notice that the $n$ partons with charge $q_{s}$ transform in the fundamental representation of the statistical $U(n)$ group. The baryonic states in the totally antisymmetric product of $n$ fundamentals are $S U(n)$ invariant, but of course transform with charge $n$ with respect to the diagonal $U(1)$ subgroup. This is the reason for the introduction of the extra parton, a singlet of $S U(n)$, and canceling the $U(1)$ charge of the 'baryon'. Finally, since the partons are treated as fermions, $n$ must be restricted to even values to ensure the correct physical interpretation of the electron bound state.

Upon integrating out the parton fields $\phi, \Phi$, as in [39], we generate various Chern-Simons actions. A purely electromagnetic Chern-Simons action with conductivity coefficient

$$
\nu=q_{d}^{2}+k n q_{s}^{2}
$$


a level- $k$ statistical Chern-Simons action for the $S U(n)$ field $\mathbf{A}-\operatorname{Tr} \mathbf{A} / n$, and an abelian ChernSimons action on the field $\operatorname{Tr} \mathbf{A} / n$ with level

$$
\kappa n=n^{2}+n k
$$

For our particular assignment of electric charges, $q_{s}=1 /(k+n), q_{d}=k /(k+n)$, we obtain a family of hydrodynamical phases with filling fraction $\nu=k /(k+n)$, realizing our noncommutative universality classes with $\kappa=k+n$. As we can see from the explicit form of the Hamiltonian, this particular linking of the abelian and non-abelian levels is borne out by imposing that the 'baryonic' partons transform in the fundamental representation of the full $U(n)$ statistical group. We can also generate all other models with $\kappa=k+s n$ by imposing irrational $U(1)$ charges with respect to the statistical field $\operatorname{Tr} \mathbf{A}$. This is much less satisfactory, since we lose the natural action of a $U(n)$ symmetry at the level of the quasiparticle, 'parton' Hamiltonian.

\section{Acknowledgements}

This work was partially supported by MCyT and FEDER under grant FPA2006-05485, CAM under grant HEPHACOS P-ESP-00346 and the European RTN network MRTN-CT-2004005104 .

\section{References}

[1] S.-C. Zhang, T. H. Hansson, and S. Kivelson, "An effective field theory model for the fractional quantum Hall effect," Phys. Rev. Lett. 62 (1988) 82-85.

[2] N. Read, "Order parameter and Ginzburg-Landau theory for the fractional quantum Hall effect," Phys. Rev. Lett. 62 (1989) 86-89.

[3] S. M. Girvin and A. H. MacDonald, "Off diagonal long range order, oblique confinement, and the fractional quantum Hall effect," Phys. Rev. Lett. 58 (1987) 1252-1255.

[4] J. Fröhlich and A. Zee, "Large scale physics of the quantum Hall fluid," Nucl. Phys. B364 (1991) 517-540.

[5] J. Fröhlich and T. Kerler, "Universality in quantum Hall systems," Nucl. Phys. B354 (1991) 369-417.

[6] A. Zee, "Quantum Hall fluids," cond-mat/9501022. Prepared for 9th Chris Engelbrecht Summer School in Theoretical Physics: Field Theory, Topology and Condensed Matter Physics, Storms River Mouth, South Africa, 17-28 Jan 1994.

[7] S. Bahcall and L. Susskind, "Fluid dynamics, Chern-Simons theory and the quantum Hall effect," Int. J. Mod. Phys. B5 (1991) 2735-2750.

[8] R. B. Laughlin, "Anomalous quantum Hall effect: An incompressible quantum fluid with fractionally charged excitations," Phys. Rev. Lett. 50 (May, 1983) 1395-1398.

[9] Z. F. Ezawa, Quantum Hall effects: Field theoretical approach and related topics. World Scientific (Singapore), 2000. 507 p. 
[10] X.-G. Wen and A. Zee, "Classification of abelian quantum Hall states and matrix formulation of topological fluids," Phys. Rev. B46 (1992) 2290-2301.

[11] B. Blok and X.-G. Wen, "Effective theories of fractional quantum Hall effect: The hierarchy construction," Phys. Rev. B42 (1990) 8145-8156.

[12] B. Blok and X.-G. Wen, "Effective theories of fractional quantum Hall effect at generic filling fractions," Phys. Rev. B42 (1990) 8133-8144.

[13] B. Blok and X.-G. Wen, "The structure of the microscopic theory of the hierarchical fractional quantum Hall effect," Phys. Rev. B43 (1991) 8337.

[14] A. López and E. H. Fradkin, "Universal structure of the edge states of the fractional quantum Hall states," Phys. Rev. B59 (1999) 15323, cond-mat/9810168.

[15] E. H. Fradkin and A. López, "Fractional quantum Hall effect and Chern-Simons gauge theories," Phys. Rev. B44 (1991) 5246-5261.

[16] G. W. Moore and N. Read, "Nonabelions in the fractional quantum Hall effect," Nucl. Phys. B360 (1991) 362-396.

[17] E. H. Fradkin, C. Nayak, A. Tsvelik, and F. Wilczek, "A Chern-Simons effective field theory for the pfaffian quantum Hall state," Nucl. Phys. B516 (1998) 704-718, cond-mat/9711087.

[18] L. Susskind, "The quantum Hall fluid and non-commutative Chern-Simons theory," hep-th/0101029.

[19] V. P. Nair and A. P. Polychronakos, "On level quantization for the noncommutative Chern-Simons theory," Phys. Rev. Lett. 87 (2001) 030403, hep-th/0102181.

[20] D. Bak, K.-M. Lee, and J.-H. Park, "Chern-Simons theories on noncommutative plane," Phys. Rev. Lett. 87 (2001) 030402, hep-th/0102188.

[21] T. Krajewski, "Gauge invariance of the Chern-Simons action in noncommutative geometry," math-ph/9810015.

[22] A. P. Polychronakos, "Noncommutative Chern-Simons terms and the noncommutative vacuum," JHEP 11 (2000) 008, hep-th/0010264.

[23] S. Hellerman and M. van Raamsdonk, "Quantum Hall physics equals noncommutative field theory," JHEP 10 (2001) 039, hep-th/0103179.

[24] A. Cappelli and M. Riccardi, "Matrix model description of Laughlin Hall states," J. Stat. Mech. 0505 (2005) P001, hep-th/0410151.

[25] A. Cappelli and I. D. Rodriguez, "Jain states in a matrix theory of the quantum Hall effect," hep-th/0610269.

[26] A. P. Polychronakos, "Quantum Hall states as matrix Chern-Simons theory," JHEP 04 (2001) 011, hep-th/0103013. 
[27] A. P. Polychronakos, "Quantum Hall states on the cylinder as unitary matrix Chern-Simons theory," JHEP 06 (2001) 070, hep-th/0106011.

[28] B. Morariu and A. P. Polychronakos, "Fractional quantum Hall effect on the two-sphere: A matrix model proposal," Phys. Rev. D72 (2005) 125002, hep-th/0510034.

[29] N. E. Grandi and G. A. Silva, "Chern-Simons action in noncommutative space," Phys. Lett. B507 (2001) 345-350, hep-th/0010113.

[30] J. L. F. Barbón and A. Paredes, "Noncommutative field theory and the dynamics of quantum Hall fluids," Int. J. Mod. Phys. A17 (2002) 3589-3606, hep-th/0112185.

[31] A. S. Schwarz, "Morita equivalence and duality," Nucl. Phys. B534 (1998) 720-738, hep-th/9805034.

[32] B. Pioline and A. S. Schwarz, "Morita equivalence and T-duality (or B versus Theta)," JHEP 08 (1999) 021, hep-th/9908019.

[33] A. Connes, M. R. Douglas, and A. S. Schwarz, "Noncommutative geometry and matrix theory: Compactification on tori," JHEP 02 (1998) 003, hep-th/9711162.

[34] L. Álvarez-Gaumé and J. L. F. Barbón, "Morita duality and large-N limits," Nucl. Phys. B623 (2002) 165-200, hep-th/0109176.

[35] D. V. Vassilevich, "Induced Chern-Simons action on noncommutative torus," hep-th/0701017.

[36] S. Elitzur, G. W. Moore, A. Schwimmer, and N. Seiberg, "Remarks on the canonical quantization of the Chern-Simons-Witten theory," Nucl. Phys. B326 (1989) 108.

[37] M. R. Douglas, "Chern-Simons-Witten theory as a topological fermi liquid," hep-th/9403119.

[38] J. M. F. Labastida, P. M. Llatas, and A. V. Ramallo, "Knot operators in Chern-Simons gauge theory," Nucl. Phys. B348 (1991) 651-692.

[39] X.-G. Wen and A. Zee, "Topological degeneracy of quantum Hall fluids," Phys. Rev. B58 (1998) 15717, cond-mat/9711223.

[40] E. Witten, "Supersymmetric index of three-dimensional gauge theory," hep-th/9903005.

[41] M. Marino and C. Vafa, "Framed knots at large N," hep-th/0108064.

[42] S. G. Naculich and H. J. Schnitzer, "Level-rank duality of the U(N) WZW model, Chern-Simons theory, and 2d qYM theory," hep-th/0703089. 